\title{
Efficient Solvent-Free Microwave Irradiation Synthesis of Highly Conductive Polypropylene Nanocomposites with Lowly Loaded Carbon Nanotubes
}

Xingru Yan, ${ }^{1}$ Jingjing Liu, ${ }^{2}$ Mojammel Alam Khan, ${ }^{3}$ Stephen Sheriff, ${ }^{4}$ Sravanthi Vupputuri, ${ }^{1}$ Rajib Das, ${ }^{5}$ Luyi Sun, ${ }^{2}$ David P. Young ${ }^{3}$ and Zhanhu Guo ${ }^{1, *}$

\begin{abstract}
It was well established that microwave radiation was utilized initially to enhance the electrical conductivity of polymer nanocomposites (PNCs) by introducing carbon nanotubes (CNTs) without using any solvent. High electrical conductivity was obtained in polypropylene (PP) nanocomposites with low CNTs loading levels. Under an inert gas protection, the CNTs were heated through the transformation of electromagnetic energy into mechanical vibrations. The surface of PP was easily molten by the heat generated by CNTs, then a well-formed CNTs network was built under proper microwave treatment period and subsequent hot pressing. CNTs loading levels and processing temperatures played an important role in the crystal structure, crystalline fraction and crystallization temperature of both nest PP and its PP/CNT PNCs. Moreover, the pressing temperature and CNT loading levels had an obvious effect on the electrical conductivity, which were the main factors on network formation. Electron transport with a three-dimensional route was observed from the study of the variable range hopping (VRH) mechanistic. The unique negative magnetoresistance (MR) phenomenon was shown in the PNCs and theoretically discussed by the forward interference model. The calculated optical band-gap of PNCs decreased with increasing the CNT loading. Other properties have been also well tested and analyzed.
\end{abstract}

Keywords: Microwave irradiation; giant magnetoresistance; electrical conductivity.

Received: 30 April 2020; Accepted: 7 July 2020.

Article type: Research article.

\section{Introduction}

Microwave irradiation is a form of electromagnetic irradiation, which is between infrared radiation and radio waves. Its wavelengths are $0.001-1 \mathrm{~m}$ with frequencies ranging from $300(0.001 \mathrm{~m})-0.3(1 \mathrm{~m}) \mathrm{GHz} \cdot{ }^{[1]}$ Microwaves have multiple applications in communications, navigation, radar technology, radio astronomy, spectroscopy and heating recourse. ${ }^{[2,3]}$ In addition, two operating frequencies of micro-waves commonly used in industrial, scientific and medical

\footnotetext{
I Integrated Composites Laboratory (ICL), Department of Chemical \& Biomolecular Engineering, University of Tennessee, Knoxville, TN 37996 USA.

2 Department of Chemical \& Biomolecular Engineering and Polymer Program, Institute of Materials Science, University of Connecticut, Storrs, CT 06269 USA.

${ }^{3}$ Department of Physics and Astronomy, Louisiana State University, Baton Rouge, LA 70803 USA.

${ }^{4}$ Institute for Advanced Composites Manufacturing Innovation, University of Tennessee, Knoxville, TN 37996, USA

${ }^{5}$ Oxea Chemical Company (OQ), Bay City, Texas 77414 USA

*E-mail: zguo10@utk.edu (Z.Guo).
}

equipment are 0.915 and $2.450 \mathrm{GHz}$ to avoid the interferences, which are hold by the Federal Communications Commission. ${ }^{[4]}$ Moreover, one of the commercially available microwave network analyzers is allowed to operate at wide frequencies from 0.1 to $20 \mathrm{GHz}{ }^{[5]}$ In 1947 , Raytheon built the first commercially available microwave oven. Over the past seven decades, the microwave oven has been an indispensable appliance in almost kitchens possessing advantages of shorter cooking time and energy saving over traditional cooking ways. Notwithstanding the microwave oven for heating food is broadly used, the application of this machine to process materials is a relatively new investigation. There are multiple benefits from microwave assisted research, including higher production quality, reduced processing times, low costs, new product development, and reduced hazards. ${ }^{[6]}$ Normally, traditional thermal processing is to transfer energy to the materials via convection, radiation, or conduction of heat through the surface of materials. During this process, the energy is transferred based on thermal gradients. However, 
microwave irradiation energy is directly transferred to materials through the interaction of microwaves with molecules, and thus displays the principle that the electromagnetic energy converts to thermal energy, rather than heat transfer. ${ }^{[7,8]}$ Hence, the molecular structure is important in the ability of the objects to interact with the microwave. Furthermore, microwaves will selectively heat the composites or multi-phase materials possessing diverse dielectric properties with a higher dielectric loss. ${ }^{[6]}$ Therefore, it is a great advantage to fabricate materials with unique and new microstructures via selectively heating distinct phases. Accordingly, microwave technology acquired fast popularity in the fabrication of metallic, ceramics and polymers based products, and composite materials..$^{[9-11]}$

Polymer nanocomposites (PNCs) have gained tremendous impetus as a result of their outstanding performances arising from their unique and special physicochemical properties such as low costs, low manufacture conditions, lightweight, and functional characteristics of nanofillers including outstanding thermal stability, robust flexibility and tunable mechanical, magnetic, rheological and electrochemical properties. ${ }^{[12-17]}$ In the rapid development of advanced materials, the PNCs with electrical conductivity or semiconductivity have drawn enormous attention because of a great deal of applications like energy storage devices, ${ }^{[18,19]}$ sensors, ${ }^{[20-22]}$ kinesiology, ${ }^{[23-25]}$ aerospace vehicles, ${ }^{[26]}$ anticorrosion coatings ${ }^{[27]}$ and microwave absorbers. ${ }^{[28-30]}$ Generally, electrically conductive PNCs are fabricated by mixing the insulative polymer matrix with conductive nanofillers. Carbon nanotubes (CNTs) are outstanding conductive nanofillers, which already have had great applications in various devices with lightweight, ${ }^{[31]}$ high aspect ratio, ${ }^{[32]}$ excellent elastic modulus, ${ }^{[33,34]}$ and great electrical and thermal conductivity. ${ }^{[35]}$ In particular, the response of CNTs to electromagnetic waves as the most attractive topic has been investigated by many researchers. Since strong absorptions are obtained with powerful outgassing, heating and light emission when CNTs are heated by microwave irradiation. ${ }^{[36]}$ In recent years, numerous researchers have used microwaves to purify and functionalize raw CNTs. ${ }^{[37,38]}$ However, the microwave irradiation used as a green technology to prepare highly electrically conductive polymer/CNT nanocomposites as engineering materials has been rarely reported so far.

In this study, we explored the efficacy of microwave irradiation as an environment-friendly method for the fabrication of electrically conductive PNCs with low loading level CNTs. The formation of CNTs network on the surface of PP granules depends on the microwave heating time, and the final electrical conductivity is related to different processing temperatures. This solvent-free preparation procedure was adjustable and repeatable. The microstructure of the PNCs was studied by both scanning electron microscope (SEM) and transmission electron microscopy (TEM). Thermogravimetric analysis (TGA) was utilized to examine the thermal stability of PNCs. The effects of CNTs on the PP were investigated by $\mathrm{X}$-ray diffraction (XRD) and differential scanning calorimeter (DSC). The rheological behaviors of the PNCs were well discussed. Moreover, the influences of CNTs loading changes and hot press temperatures on the electrical conductivity, magnetoresistance, and optical property were studied for all PNCs. The electrical conductivity mechanism was well theoretically analyzed by the variable range hopping model.

\section{Experiments \\ 2.1 Materials}

Polymer matrix polypropylene (PP) was supplied from the Total Company (Total 3270, $M_{w}:$ 297000, melt flow rate (MFR): $2.0 \mathrm{~g} 10 \mathrm{~min}^{-1}$, density: $\left.0.91 \mathrm{~g} \mathrm{cc}^{-1}\right)$. The carbon nanotubes (CNTs, SWeNT SMW 200X) were provided by SouthWest Nano Technologies, Inc with $10.4 \mathrm{~nm}$ average diameter and $4.3 \mu \mathrm{m}$ average length. No further treatment was applied to all the chemicals utilized in this project.

\subsection{Nanocomposites Preparation}

The PP/CNTs nanocomposites mixed CNTs with loadings of $0.15,0.50,1.20$ and $1.30 \mathrm{wt} \%$ were prepared by microwave solvent-free irradiation as the following procedure. As shown in Scheme 1, $125 \mathrm{~mL}$ Buchner flask containing $5 \mathrm{~g}$ PP and $2 \mathrm{~g}$ CNTs was purged by nitrogen for $15 \mathrm{~min}$, then the vents were sealed by the parafilm immediately. The PP granules were fully surrounded by CNTs after mechanically shaking about 30 s (Vortex-Genie 2, Gear: 6). After that, the PP/CNTs mixture was microwaved (Rival 700 Watts, $2.450 \mathrm{GHz}$ ) for 5 $\mathrm{s}$ as a period; during each interval, the mixture was taken out and mechanically shaken for $3 \mathrm{~s}$ (Gear: 6). The samples were collected after 4, 5, 6, and 7 periods and marked as 20, 25, 30 and $35 \mathrm{~s}$, respectively. Afterward, the microwave treated mixture was poured into a designed container with ordered pores smaller than granules on the inner wall to collect surplus CNTs (Gear: 8, 3 min). The pure PP was treated as the same procedure without CNTs, which was marked as m_Pure PP. The finally collected pellets were shaped into a disk (diameter: $25 \mathrm{~mm})$ with a series of $\left(140,160\right.$ and $\left.180^{\circ} \mathrm{C}\right)$.

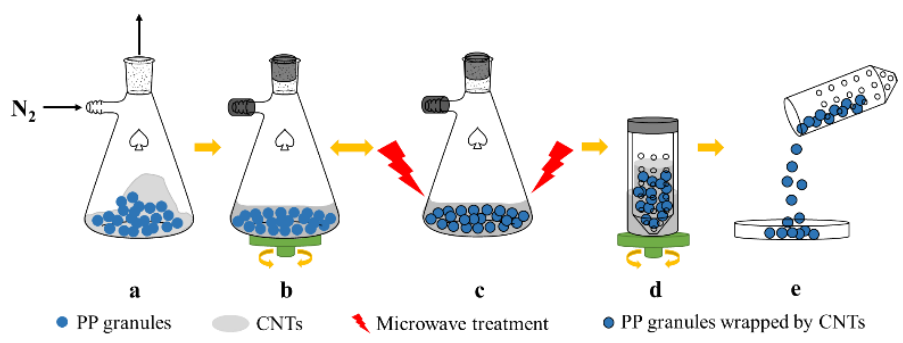

Scheme 1. The procedures of PP/CNTs PNCs fabrication by the microwave solvent-free irradiation. 

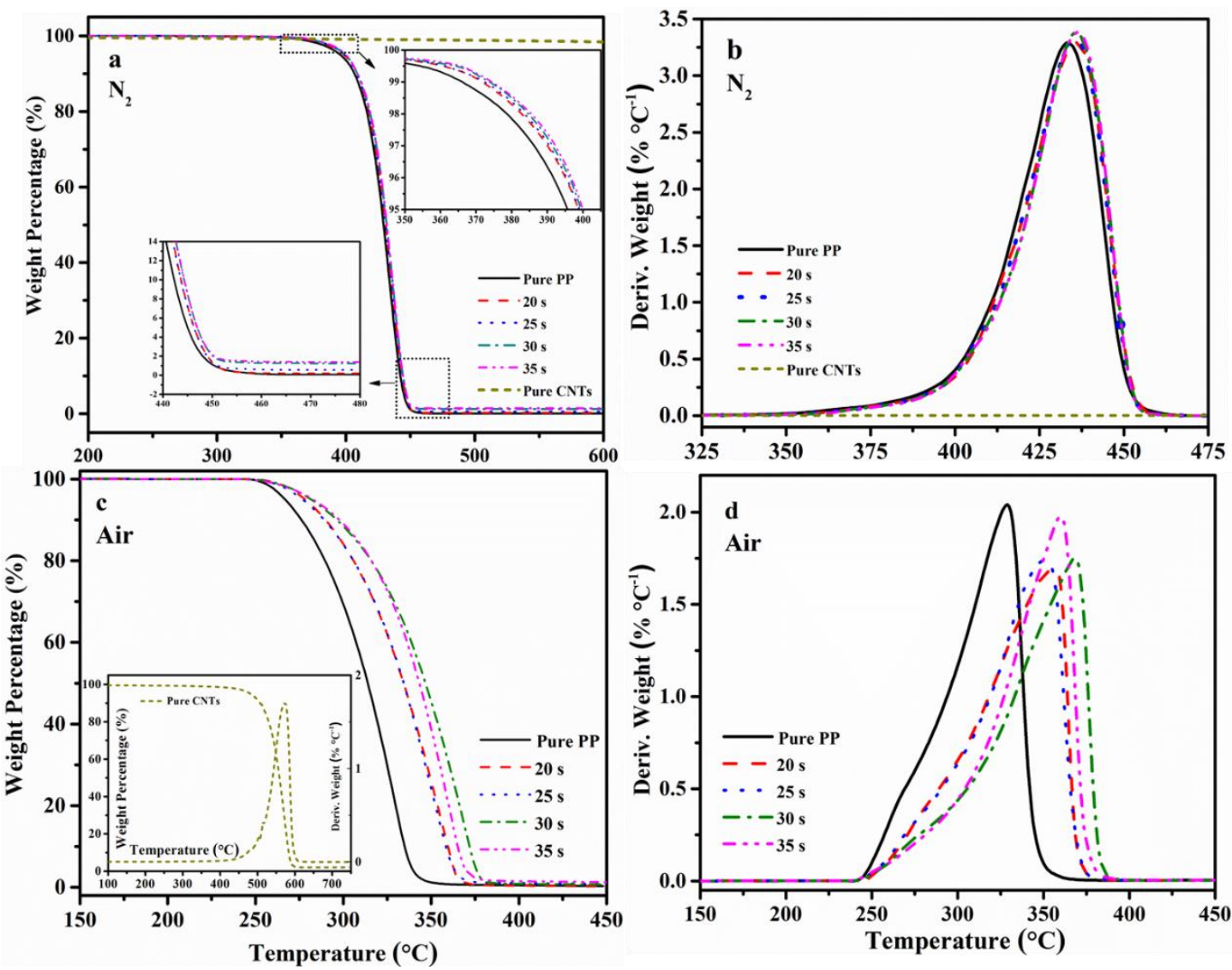

Fig. 1 Thermogravimetric analysis (TGA) curves of pure PP and its PP/CNT PNCs under 20, 25, 30, 35 s microwave treatment under (a) $\mathrm{N}_{2}$ and (c) air conditions; and the corresponding derivative thermogravimetric (DTG) (b\&d); Insert in (c): TGA and DTG curves of neat CNTs.

\subsection{Characterizations}

The melting and crystallization properties of pure PP and nanocomposites were analyzed by a TA Instruments Q2000 differential scanning calorimeter (DSC). This test was completed in a nitrogen environment with a flow rate of about $50 \mathrm{~mL} \mathrm{~min}^{-1}$ and a heating rate of $10{ }^{\circ} \mathrm{C} \mathrm{min}^{-1}$. These samples were about $10 \mathrm{mg}$ for each test. During the test, each sample was heated from 30 to $200{ }^{\circ} \mathrm{C}$ at first to get rid of the thermal history, then lower temperate to $30^{\circ} \mathrm{C}$, and reheated again to $200^{\circ} \mathrm{C}$. The data of the first cooling and second heating steps were collected to be analyzed. Thermogravimetric analysis (TGA, TA Instruments Discovery) was used to study the thermal stability of the PP/CNTs PNCs. It was carried out from 30 to $600{ }^{\circ} \mathrm{C}$ under both air and nitrogen atmospheres at $10{ }^{\circ} \mathrm{C}$ $\min ^{-1}$ heating rate.

The rheometer (TA Instruments Discovery HR-1) examined the storage and loss modulus and loss factor of the $\mathrm{PP} / \mathrm{CNTs}$ PNCs. An environmental test chamber (ETC) aluminum parallel-plate geometry (25 $\mathrm{mm}$ in diameter) was utilized to perform the dynamic rheological measurements with the dynamic oscillation frequency $0.1-100 \mathrm{rad} \mathrm{s}^{-1}$ at $1.0 \%$ strain. The viscosity measurements were completed from 0.1 to $100 \mathrm{~s}^{-1}$ of shear rate at $220{ }^{\circ} \mathrm{C}$. The PP/CNTs test samples were shaped into disc pellets with a diameter of $25 \mathrm{~mm}$ and the average thickness was about $2.0 \mathrm{~mm}$ through applying a clamping force of 5 Tons in a benchtop auto presser (Carver Standard Auto $\mathrm{CH}$ ).

The crystalline structures of pure PP and its PNCs were examined by X-ray diffraction (XRD) scanning from 10 to $30^{\circ}$. The morphology was investigated by emission scanning electron microscope (SEM, Zeiss MERLIN). The microstructure of CNTs and nanocomposites from the fracture surface of the samples was observed. Meanwhile, the fracture surface was created in liquid nitrogen for characterization.

UV/VIS/NIR spectrophotometer (JASCO, Model V-670) accompanied with a Jasco ISN-723 diffuse reflectance accessory was applied to test Ultraviolet-visible (UV-vis) diffuse reflectance spectra (DRS) of the PP/CNTs PNCs.

The electrical resistivity was studied using a standard four-probe method. The samples used in this measurement were shaped into disc pellets by hot presser following the same procedure as the samples used in the viscosity measurements. The magnetoresistance (MR) was measured in a 9-Tesla Physical Properties Measurement System (PPMS) by Quantum Design at $290 \mathrm{~K}$, which was carried out using a standard four-probe technique.

\section{Results and Discussion}

\subsection{Thermal stability}

The thermal decomposition data of pure PP and its PNCs at both nitrogen and air conditions are shown in Fig. 1a\&c; and the corresponding derivative weight loss curves are presented 
in Fig. 1b\&d, respectively. The CNT loadings of PP/CNT PNCs under different microwave periods are summarized in Table 1 .

Table 1. TGA record of the pure PP and its PNCs with CNTs.

\begin{tabular}{l|l|lllll}
\hline \multirow{2}{*}{$\begin{array}{c}\text { CNT } \\
\text { loading } \\
\text { (wt.\%) }\end{array}$} & \multicolumn{2}{c}{ Pure PP } & $20 \mathrm{~s}$ & $25 \mathrm{~s}$ & $30 \mathrm{~s}$ & $35 \mathrm{~s}$ \\
\cline { 2 - 7 } & \multicolumn{2}{c}{0} & 0.15 & 0.50 & 1.20 & 1.30 \\
\hline \multirow{2}{*}{$\mathrm{N}_{2}$} & $T_{\text {on }\left[{ }^{\circ} \mathrm{C}\right]}$ & 414.26 & 415.98 & 416.18 & 417.10 & 417.22 \\
& $T_{\text {end }}\left[{ }^{\circ} \mathrm{C}\right]$ & 444.36 & 445.96 & 445.62 & 445.93 & 446.05 \\
\hline \multirow{2}{*}{ Air } & $T_{\text {on }}\left[{ }^{\circ} \mathrm{C}\right]$ & 290.42 & 306.169 & 306.27 & 320.12 & 319.41 \\
& $T_{\text {end }}\left[{ }^{\circ} \mathrm{C}\right]$ & 339.26 & 365.121 & 363.34 & 377.13 & 369.31 \\
\hline
\end{tabular}

For PNCs under $\mathrm{N}_{2}$ atmosphere, there is a pointy weight loss stage in the temperature range from 400 to $450{ }^{\circ} \mathrm{C}$, which is consistent with the previous observation, ${ }^{[39]}$ owing to a large scale thermal degradation of PP polymer chains. The onset degradation temperatures $\left(T_{o n}\right)$ and the temperatures of the maximum weight loss $\left(T_{\text {end }}\right)$ of CNTs/PP PNCs increase slightly with increasing CNTs loading as shown in Table 1. This indicates the stabilization of PP by the introduced CNTs. This result also can be proved by DTG curves, in which the peaks of PP/CNT PNCs move to higher temperatures than that of pure PP (Fig. 1b). The CNT loadings of PP/CNT PNCs treated under a series of microwave periods can be determined by the TGA curves under $\mathrm{N}_{2}$ condition, which are $0,0.15,0.5$, 1.20, 1.30 wt.\% CNTs for pure PP and 20, 25, 30 and $35 \mathrm{~s}$ microwave heating PP/CNTs PNCs, respectively.

$\mathrm{PP} / \mathrm{CNT}$ PNCs with $1.20 \mathrm{wt} \% \mathrm{CNTs}$ have the highest $T_{\text {on }}$ $\left(320.12^{\circ} \mathrm{C}\right)$ under air condition as shown in Table 1 , which is about $30^{\circ} \mathrm{C}$ higher than that of pure PP. The hardy interfacial particle-polymer interactions is one of the reasons. ${ }^{[40]}$ Another reason is that the heat is absorbed by neat CNTs transitorily and thus postpones the degradation of PP chains since the neat CNTs begin to be decomposed at around $500{ }^{\circ} \mathrm{C}$, which was observed from TGA of neat CNTs in the insert of Fig. 1c. However, when the loading of CNTs comes to $1.30 \mathrm{wt} \%$, the $T_{\text {on }}$ decreases slightly. Since heat transfers to polymer more deeply and quickly with a good thermal conductivity of mixed CNTs. Therefore, the network structure is destroyed rapidly in the polymer matrix by increasing the CNTs loading. The above phenomena also can be obtained in DTG curves as shown in Fig. 1d.

\subsection{Melting and crystalline behaviors}

The melting and crystallization behaviors of pure polymer and its PNCs can be depicted using the DSC endothermic and exothermic data as presented in Fig. 2, respectively. The exact crystallization data $\left(T_{c}\right.$ : crystalline peak temperature; $\Delta H_{c}$ : crystallization enthalpy; $T_{m}$ : melting peak temperature; $\Delta H_{m}$ : fusion enthalpy; and $F_{c}$ : crystalline fraction) are shown in Table 2. The $T_{c}$ and $T_{m}$ are easily read from curves. The $\Delta H_{c}$ and $\Delta H_{m}$ data are simply integrated from the DSC curves.
Compare crystallization and fusion enthalpy, the $\Delta H_{c}$ is smaller than the $\Delta H_{m}$ value for all samples, which is attributed to the sample recrystallization. The $F_{c}$ for pure polymer and nanocomposites is calculated from Equation $1:[41]$

$$
F_{c}=\frac{\Delta H_{m}}{\Delta H_{m}^{0} \times(1-x)}
$$

where $\Delta H_{m}^{0}$ is the enthalpy for a theoretical $100 \%$ crystalline polymer. As a reference, PP is $209 \mathrm{~J} \mathrm{~g}^{-1[42]}$ and $x$ is CNTs loadings. The as-received (pure PP) and the treated (m_Pure PP) PP granules are compared in Fig. 2a to reveal the effects of microwave on the PP granules. The cooling and heating curves of these two samples almost overlap with closed $T_{c}$ and $T_{m}$ values, and the $F_{c}$ also stays unchanged. Therefore, it confirms that the microwave has no influence on pure PP granules after 35-s microwave treatment. Since no or negligible energy absorption was obtained in the pure PP granules with a very low dielectric loss factor. Consequently, microwave energy does not work on them. ${ }^{[43]}$

Table 2. DSC paraments of pure PP and PNCs with different microwave treatments.

\begin{tabular}{lccccc}
\hline Treatment Time & $\begin{array}{c}T_{c} \\
\left({ }^{\circ} \mathrm{C}\right)\end{array}$ & $\begin{array}{c}\Delta H_{c} \\
\left(\mathrm{~J} \mathrm{~g}^{-1}\right)\end{array}$ & $\begin{array}{c}T_{m} \\
\left({ }^{\circ} \mathrm{C}\right)\end{array}$ & $\begin{array}{c}\Delta H_{m} \\
\left(\mathrm{~J} \mathrm{~g}^{-1}\right)\end{array}$ & $\begin{array}{c}F_{c} \\
(\%)\end{array}$ \\
\hline Pure PP & 115.71 & 154.0 & 167.33 & 124.2 & 59.43 \\
m_Pure PP & 115.19 & 154.2 & 167.70 & 124.0 & 59.33 \\
$20 \mathrm{~s}$ & 113.38 & 151.0 & 169.45 & 130.6 & 62.58 \\
$25 \mathrm{~s}$ & 114.29 & 150.7 & 168.55 & 131.0 & 62.99 \\
$30 \mathrm{~s}$ & 113.36 & 150.2 & 168.14 & 132.1 & 63.97 \\
$35 \mathrm{~s}$ & 113.29 & 151.8 & 167.88 & 133.8 & 64.86 \\
$30 \mathrm{~s} \_140^{\circ} \mathrm{C}$ & 113.55 & 151.4 & 167.81 & 127.6 & 61.79 \\
$30 \mathrm{~s} \_160^{\circ} \mathrm{C}$ & 114.69 & 152.9 & 167.22 & 129.3 & 62.62 \\
$30 \mathrm{~s} 180^{\circ} \mathrm{C}$ & 114.70 & 153.6 & 165.59 & 129.4 & 62.67 \\
\hline
\end{tabular}

The microwave treatment duration of PP/CNT PNCs was studied in Fig. 2b. The $T_{c}$ values of PP/CNT PNCs are smaller than those of pure PP. The variation of $T_{c}$ is caused by different roles of the CNTs during crystallization process of PP, which can be explained by the spherulitic growth rate $(G)$ as shown by Equation $2:{ }^{[44-46]}$

$$
G=G_{0} \exp \left[\frac{-U^{*}}{R\left(T_{c}-T_{\infty}\right)}\right] \exp \left\{\frac{-K_{g}}{\left[T_{c} f\left(T_{m}^{0}-T_{c}\right)\right]}\right\}
$$

where $G_{0}$ is a factor on the units $\mathrm{cm} \mathrm{s}^{-1}, U^{*}$ is the activation energy for the alteration of polymer chain segments to the crystallization sites, $T_{m}^{0}$ is the equilibrium $T_{m}, R$ is the gas constant, $f$ is the factor which showed the decrease in heat of fusion with decreasing temperature, $T_{\infty}$ is the hypothetical temperature where all the motions associated with viscous flow are ceased, the $K_{g}$ is the nucleation constant, which can be presented by Equation 3:[45] 


$$
K_{g}=\frac{4 E E_{e} b_{0} T_{m}^{0}}{\Delta h_{f} \rho_{c} k_{B}}
$$

where $E$ and $E_{e}$ are side and fold surface free energies that measure the work required to create a new surface, $b_{0}$ is the single layer thickness, $\Delta h_{f} \rho_{c}$ is the enthalpy of melting and $k_{B}$ is the Boltzmann constant. Hence, the crystallization formation is affected by two parts as shown in Equation 2, the transport of polymer chain segments to the growth front contributes to the first part and the second part is for the nucleation process. After introducing CNTs on the surface of pure PP granules, the CNTs would immobilize partial polymer chains to the growth front, which results in $U^{*}$ value increasing and leads the crystallization temperature $\left(T_{c}\right)$ to decrease. ${ }^{[47]}$ However, the CNTs can be acted as nucleation sites and foster the crystallization of PP by diminishing the surface free energy barrier $\left(E, E_{e}\right)$ towards nucleation. Therefore, the crystallization of PP/CNT PNCs under $25 \mathrm{~s}$ microwave treatment with $0.5 \mathrm{wt} \% \mathrm{CNTs}$ was at a slightly higher temperature at the beginning. ${ }^{[48]}$ In addition, a small shoulder can be obtained in PNCs with 1.20 and $1.30 \mathrm{wt} \%$ CNTs. However, pure PP and PNCs with 0.15 and $0.50 \mathrm{wt} \%$ CNTs have no shoulder during the crystallization process. The separation of crystallization peaks depicts a variation of the crystal structures. Both $\alpha$ and $\gamma$ phases of PP were generated

during crystallization. Meanwhile, the $\gamma$ phase can be formed with a lower supercooling. During the cooling proceeding, $\gamma$ phase PP would appear dominant lamellae at first, then the $\alpha$ phase would form on it. ${ }^{[49]}$ Thus, the small shoulder is produced owning to $\gamma$ phase formation of PP by cooling and the $\gamma$ phase PP promoted by CNTs.

In the interest of studying the effects of hot-pressing process temperature on PP/CNTs PNCs, PNCs treated by $30 \mathrm{~s}$ microwave and hot pressed at 140,160 and $180{ }^{\circ} \mathrm{C}$ were investigated. The results are shown in Fig. 2c. From the data summarized in Table 2, the $F_{c}$ increases slightly after introducing CNTs into the PP host. The higher $F_{c}$ of these PNCs is due to a higher process temperature which promotes the crystalline structure formation of the PP chains.

\subsection{Crystallinity}

The effects of the CNT loading and hot press temperature on the crystalline structure of pure PP and its PNCs were detected by XRD. PP possesses a noteworthy complexity of crystal structures including $\alpha, \beta$ and $\gamma$ phases, though the simplicity of its chemical structure. Each of $\alpha, \beta$ and $\gamma$ phase shows its own characteristic peaks in the XRD patterns. As presented in Fig. 3 , the peak intensity of pure PP increases gradually with increasing processing temperature, which is concordant with Zanneti's results. ${ }^{[50]}$
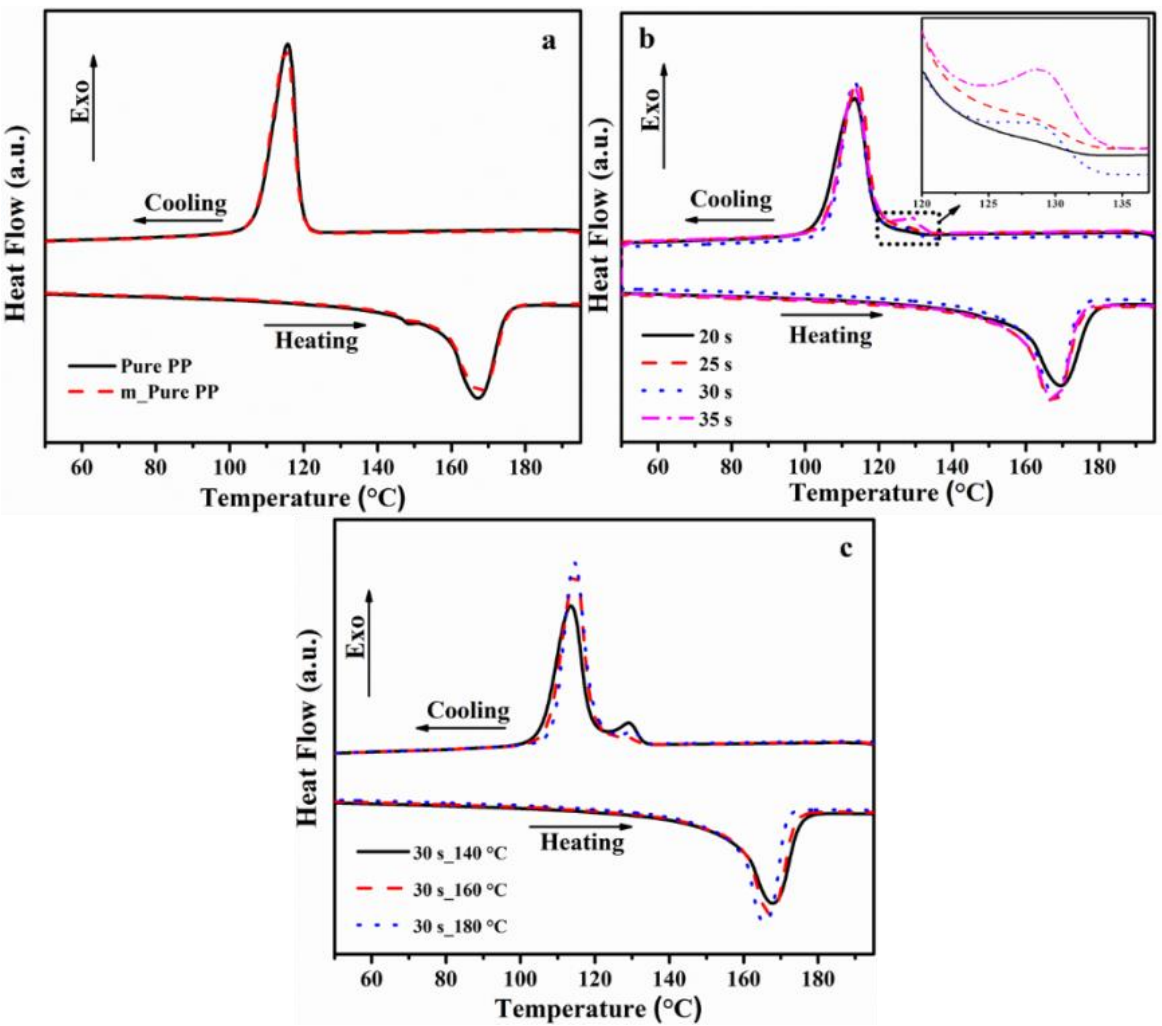

Fig. 2 DSC curves of a. pure PP granules with (m_Pure PP) and without (Pure PP) 35 s microwave treatment; b. CNTs wrapping PP granules microwave treated by $20 \mathrm{~s}, 25 \mathrm{~s}, 30 \mathrm{~s}$ and $35 \mathrm{~s}$, respectively; c. CNTs/PP granules with $30 \mathrm{~s}$ microwave treatment processed at 140,160 and $180^{\circ} \mathrm{C}$, respectively. 


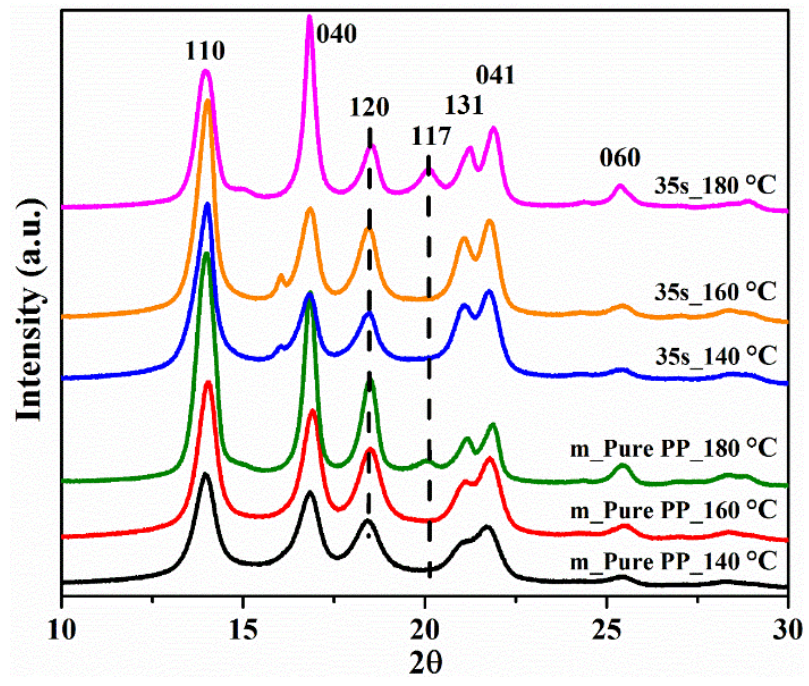

Fig. 3 X-ray diffraction curves of pure $\mathrm{PP}$ and $35 \mathrm{~s}$ microwave heating PP/CNTs PNCs processed at 140,160 and $180^{\circ} \mathrm{C}$.

However, the peak intensity of PP/CNT PNCs with $35 \mathrm{~s}$ microwave heating increases firstly at $160{ }^{\circ} \mathrm{C}$, then both peak intensity and crystal structures undergo changes when processing temperature reaches $180{ }^{\circ} \mathrm{C}$. The intensity of the (040) peak is stronger than that of the (110) peak, and the characteristic peak of $\gamma$ phase is obtained at $2 \theta=20.09^{\circ}$. The peaks at $2 \theta=14.04,16.85,21.13,21.92$ and $20.50^{\circ}$ correspond to the (110), (040), (131), (041) and (060) crystalline planes of $\alpha-\mathrm{PP}$, respectively. The $\alpha$ phase is usually determined from the peak $2 \theta=18.50^{\circ}$ corresponding to the 120 crystalline plane. ${ }^{[51]}$ To investigate the effects of CNTs on the crystalline phase of PP, the quantity of $\gamma$ phase $\operatorname{PP}\left(X_{\gamma}\right)$ can be calculated by Equation (4): ${ }^{[52]}$
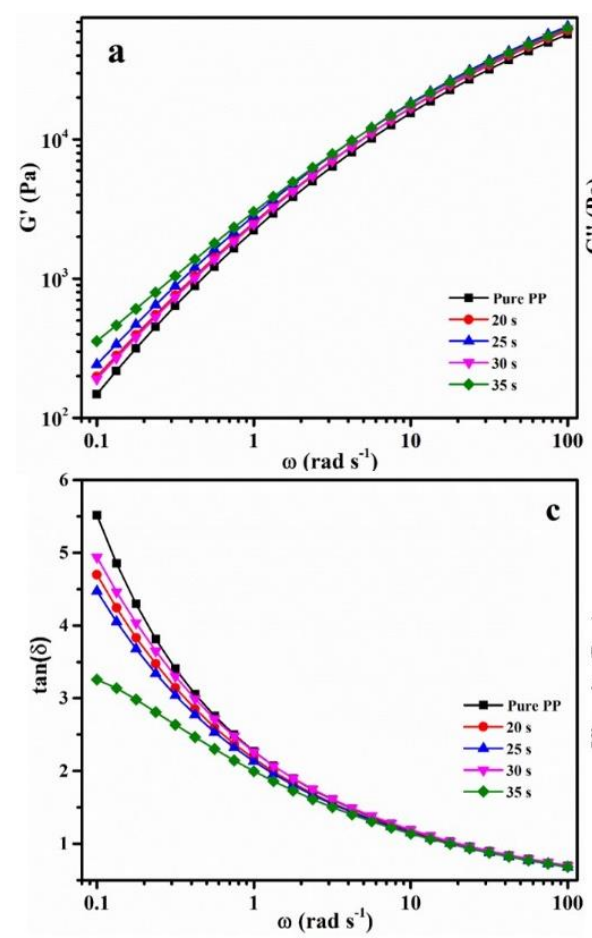

$$
X_{\gamma}=h_{\gamma} /\left(h_{\gamma}+h_{\alpha}\right)
$$

where $h_{\alpha}$ and $h_{\gamma}$ are the peak height at $2 \theta=18.50$ and $20.09^{\circ}$ for the (120) and (117) peak, respectively. The values of $X_{\gamma}$ are 0.1197 and 0.3676 for pure $\mathrm{PP}$ and $35 \mathrm{~s}$ microwave heating $\mathrm{PP} / \mathrm{CNT}$ PNCs pressed at $180^{\circ} \mathrm{C}$, respectively. The PNCs with $35 \mathrm{~s}$ microwave exhibit more amount of $\gamma$ phase PP compared with pure PP, which confirms that the CNTs advanced the $\gamma$ phase PP formation.

\subsection{Melt rheological behaviors}

The melt rheological behavior of PNCs provides important information for evaluating their internal structures including the fillers dispersion, aspect ratio, orientation of fillers, and the interaction between fillers and the polymer matrix. ${ }^{\left[{ }^{[3]}\right]}$ Fig. 4(ac) presents the trends of storage modulus $\left(G^{\prime}\right)$, loss modulus $\left(G^{\prime \prime}\right)$ and loss factor $(\tan \delta$ ) for pure PP and its PNCs processed at $180{ }^{\circ} \mathrm{C}$ at a range of angular frequency $(\omega)$. The full relaxation is observed among the PP chains, which reveals a typical homogeneous liquid-like terminal behavior. $G^{\prime}$ is relatively scattered at the low frequency range, which is due to the increased solid-like behavior of the PNCs after introducing CNTs. Meanwhile, similar phenomenon is also obtained in the PP/MWCNT (multi-walled carbon nanotube) PNCs fabricated by diluting highly concentrated masterbatch chips. ${ }^{\left[{ }^{44]}\right.}$ However, the influence of the CNTs loading on the viscoelastic properties $\left(G^{\prime}, G^{\prime \prime}\right.$ and $\tan \delta$ ) of PNCs is relatively weak at high frequencies, revealing that the local dynamics of the PP chains can be affected by the CNTs effectively. ${ }^{[55]}$
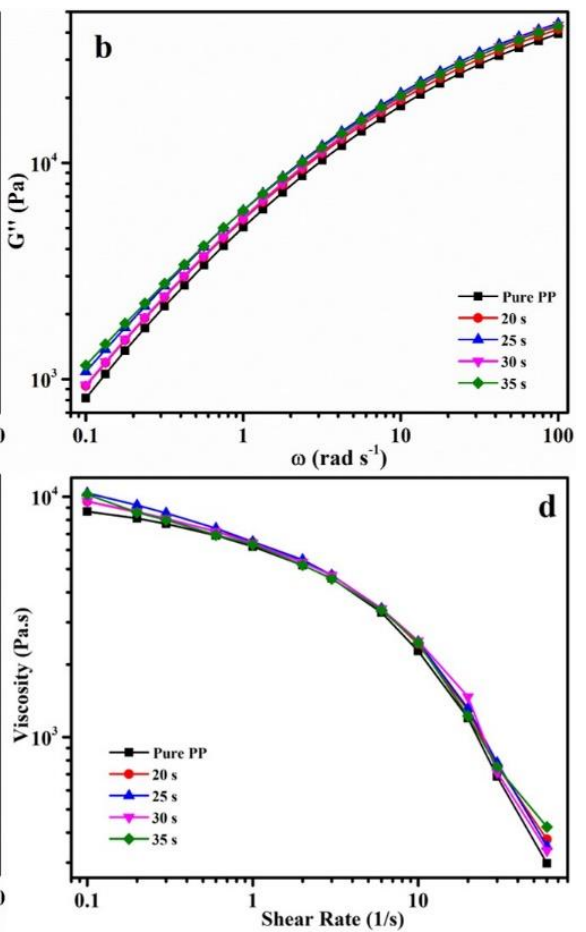

Fig. 4 (a) Storage modulus $\left(G^{\prime}\right)$, (b) loss modulus $\left(G^{\prime \prime}\right)$, (c) loss factor $(\tan \delta$ ) vs. angular frequency $(\omega)$ and (d) viscosity $v s$. shear rate of pure $\mathrm{PP}$ and $\mathrm{PP} / \mathrm{CNT}$ nanocomposites with different time microwave treatment processed at $180{ }^{\circ} \mathrm{C}$. 
In addition, $G^{\prime \prime}$ curve has a similar tendency to linearly increase with increasing frequency. The $\tan \delta$ (the ratio of $G^{\prime \prime}$ to $G^{\prime}$ ) is employed to evaluate the PNCs' damping property. Compared with PP/CNT PNCs, the tan $\delta$ value of pure PP is slightly higher. This is because that the polymer chain relaxation and relative movement of PP/CNT PNCs are hardly hampered by the introduced CNTs, which results in less generation of internal chain-chain friction heat in PNCs than that in pure PP when applying the same oscillation frequency. The viscosity of pure PP and its PNCs was also studied and shown in Fig. 4d at a range of shear rate. All these samples did not present a Newtonian plateau and showed shear thinning behaviors under melting state, which displayed a decreasing viscosity with increasing shear rate. ${ }^{[56]}$ Random thermal motion restrains the alignment of CNTs. Meanwhile, the speed of recreating of polymer chain entanglement is slower than that of breaking the physical cross-linked sites with increasing the shear rate. The viscosity of PNCs with the incorporation of CNTs is slightly larger than that of pure PP, which results in the increased melt free volume after adding nanofillers in the entangled and confined systems, and the mobility of polymer chains was restrained due to the dilution effect from nanoparticles. ${ }^{[57]}$ The stronger shear thinning behavior was also obtained in PP/CNT PNCs made by the masterbatch dilution method. ${ }^{[58]}$

\subsection{Formation mechanism of PP/CNT PNCs}

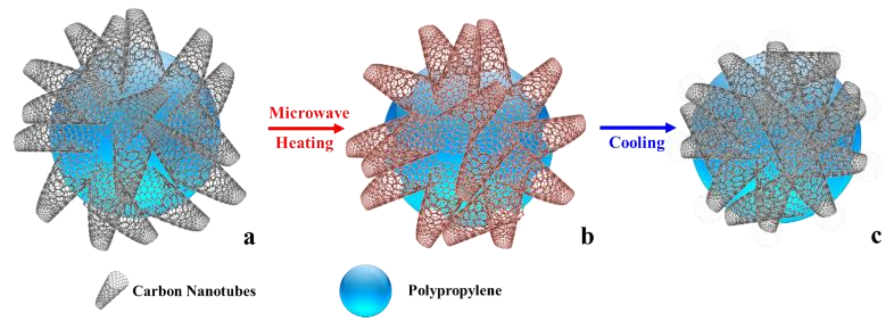

Scheme 2. The mechanism of PP/CNTPNCs fabrication via microwave irradiation.

A fundamental understanding of the reactions of materials with microwave irradiation is the key to develop the technology for widespread and deep use. The mechanism of $\mathrm{PP} / \mathrm{CNT}$ PNCs prepared by microwave irradiation is presented in Scheme 2. The PP granules were fully covered by CNTs in an inert atmosphere. Based on electrostatic forces, the PP granules were also wrapped by slight CNTs as shown in Scheme 2a. With the action of microwave on the CNTs and polymer mixture, the heat was generated in CNTs without any effect on PP granules as presented in Scheme 2b. Then, the temperature of CNTs was controlled high enough to melt the surface of PP granules without the polymer decomposition by proper heating period. To prepare homogenously coating materials, the mixture was necessarily shaken well during the nanocomposites preparation since the microwave resource was localized. Consequently, the CNTs were stuck on the PP granules surface and form a network as shown in Scheme 2c.
The heat caused by microwave irradiation can be explained by two main factors as conduction and dipolar polarization. Normally, different impurities can be obtained in commercially available CNTs such as carbon and metals, which are electrically conductive and can be explained by the mechanism based on conduction heating. For this mechanism, the electric field of microwaves makes the electrons move, which causes samples to be heated instead of heat conduction. In this way, the metal impurities should cause localized superheating by inducing Joule heating. ${ }^{[59]}$ Moreover, the extended $\pi$-system allows conductivity to work on localized heating. ${ }^{[00]}$ Meanwhile, the generation of gas plasma from absorbed gases (particular $\mathrm{H}_{2}$ ) in CNTs should be considered as another potential way of localized superheating under microwave irradiation. ${ }^{[36]}$ The dipolar polarization also has a contribution to imperfect CNTs microwave heating due to the structural imperfections of as-synthesized CNTs to cause the ballistic transport's decay, which results in Joule heating representing as superheating. ${ }^{[61]}$ In 2006, Ye et al. provided an easy model to explain microwave heating of CNTs describing the transformation of electromagnetic energy into mechanical vibrations. ${ }^{[62]}$ This model emphasized that a transverse parametric resonance causes the CNTs undergoing superheating, which is a consequence of the polarization of CNTs exposed in the microwave radiation.

3.6 Electrical conductivity ( $\sigma$ ) of PP/CNTs nanocomposites The conductivity $(\sigma)$ of the pure PP and its PNCs processed at different processing temperatures as a function of the CNT loadings is shown in Fig. 5.The $\sigma\left(\sim 10^{-9} \mathrm{~S} \mathrm{~cm}^{-1}\right)$ of pure PP was acquired from reference. ${ }^{[63]}$ It is obtained that the $\sigma$ elevated with increasing the CNT loadings. Meanwhile, the PNCs with 1.30 wt.\% CNTs processed at $160{ }^{\circ} \mathrm{C}$ show the highest $\sigma$ of $0.46 \mathrm{~S} \mathrm{~cm}^{-1}$.

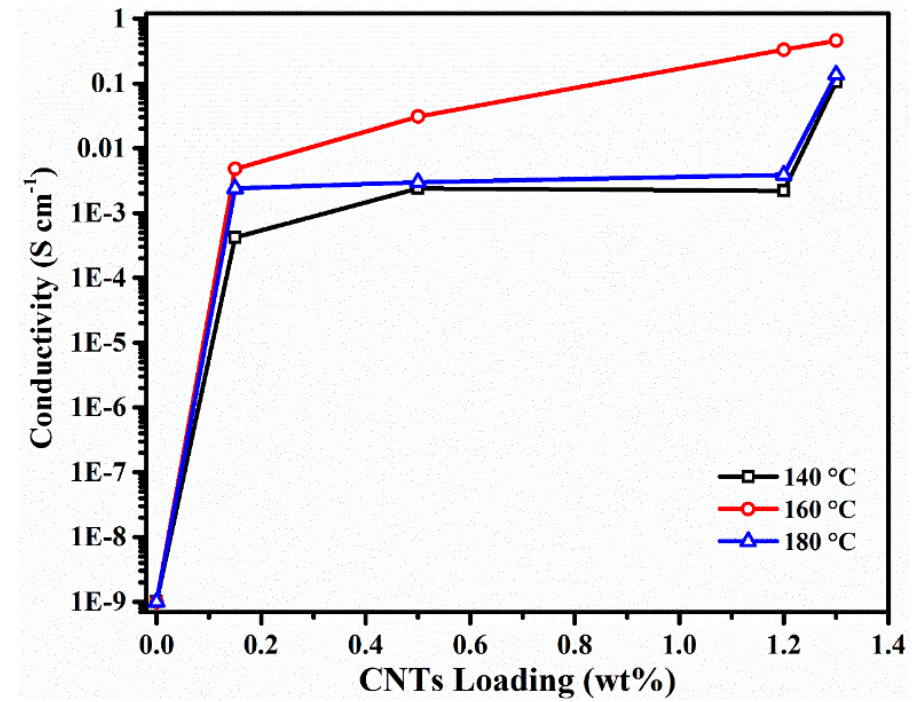

Fig. 5 Electrical conductivity $(\sigma)$ of PNCs as a function of CNTs weight loadings for the samples processed at 140,160 and $180^{\circ} \mathrm{C}$. 

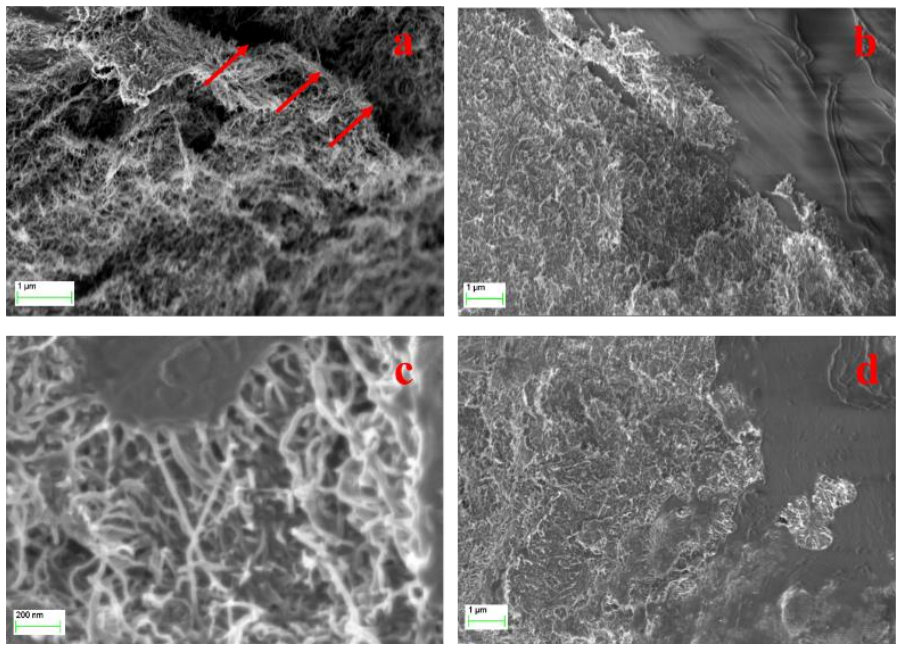

Fig. 6 SEM microstructures of PP/CNT PNCs with (a) $25 \mathrm{~s}$ microwave treatment prepared at $140{ }^{\circ} \mathrm{C}$, (b) $20 \mathrm{~s}$ microwave treatment prepared at $160{ }^{\circ} \mathrm{C}$, and $35 \mathrm{~s}$ microwave treatment prepared at (c) 160 and (d) $180{ }^{\circ} \mathrm{C}$.

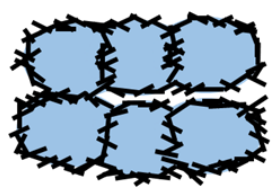

a

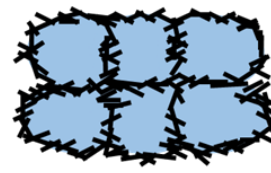

b

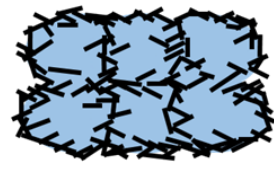

c $\checkmark$ Carbon Nanotubes

Polypropylene

Scheme 3. Morphology of PNCs with CNTs at different processing temperature.

Recently, Wu et al. reported that the $\sigma$ of PP/MWCNT composites can reach $\sim 10^{2} \mathrm{~S} \mathrm{~cm}^{-1}$ with $10 \mathrm{wt} \%$ MWCNTs prepared by mixing mothed. However, the $\sigma$ is less than $\sim 10^{-6}$ $\mathrm{S} \mathrm{cm}^{-1,}$ when the composites contained $\sim 2 \mathrm{wt} \%$ MWCNTs. ${ }^{[64]}$ Compared with materials fabricated by conventional method, the higher $\sigma$ examined in this study was attributed to the wellformed conductive CNT network with advantages of microwave heating and processing temperature. With different microwave heating periods, the PP/CNT PNCs were coated with different weights of CNTs, which was already proved by TGA. As shown in Scheme $3(\mathrm{a})$, at $140^{\circ} \mathrm{C}$, the PP pellets just tend to melt. The pellets could only change shapes without damaging the CNT layer during the hot press process. But the interactions among each pellet are weak to make each CNTs layer combine together. As red arrows marked in Fig. 6a, a gap can be obtained between two pellets coated with CNTs, which arises from the less mobility of both CNTs and PP chains at $140{ }^{\circ} \mathrm{C}$. That is the reason that $\mathrm{PP} / \mathrm{CNT}$ PNCs processed at $140{ }^{\circ} \mathrm{C}$ have the smallest $\sigma$ at each nanofiller content. At $160^{\circ} \mathrm{C}$, the pellets were in melting state, and the interactions of pellets were enhanced by the easier movement of CNTs and PP chains, which resulted in CNTs layers forming tightly network as presented in Scheme 3(b). That can be confirmed by Fig. $6 \mathrm{~b}$, in which the CNTs cling more closely on the surface of PNCs than that shown in Fig 6a. The higher processing temperature leads to less resistance to molecular motions and strong interactions between nanofiller and polymer chains. ${ }^{[65]}$ Moreover, with a longer microwave treatment, a better network was constructed as shown in Fig. 6c. The $\mathrm{PP} / \mathrm{CNT}$ PNCs processing at $160{ }^{\circ} \mathrm{C}$ have the highest $\sigma$ and remain an up-trend. The electrons could hop from a nanotube to an adjacent one in the formed CNTs network structure, which results in the enhanced electrical conductivity.[66] However, when the temperature reached $180{ }^{\circ} \mathrm{C}$, the $\sigma$ became lower, owing to the reduced density of CNTs network, even the network of CNTs was broken as viewed in Scheme 3(c). The PNCs were totally molten at $180^{\circ} \mathrm{C}$, letting molecules move continually. Thus, more CNTs were released from the layers. From Fig. 6d, the surface became flatter and the CNTs coating layer was destroyed by PP since in the molten matrix, only a part of CNTs can participate in the conductive path formation. Then, the morphology of the PNCs was also investigated by TEM, as shown in Fig. 7. The dispersed CNTs observed among the PP matrix formed the connected structures. However, the CNT density of PNCs with $20 \mathrm{~s}$ microwave treatment prepared at $140{ }^{\circ} \mathrm{C}$ is lower than that of PNCs with $30 \mathrm{~s}$ microwave treatment prepared at $160{ }^{\circ} \mathrm{C}$, which is the main reason that PNCs with $30 \mathrm{~s}$ microwave treatment processed at $160{ }^{\circ} \mathrm{C}$ exhibit the highest $\sigma$.

\subsection{Electrical conductivity mechanism}

The resistivity as a function of temperature for PNCs with 20 s microwave treatment prepared at $180^{\circ} \mathrm{C}, 30 \mathrm{~s}$ microwave treatment prepared at $160{ }^{\circ} \mathrm{C}$, and $35 \mathrm{~s}$ microwave treatment prepared at 140 and $180{ }^{\circ} \mathrm{C}$ is presented in Fig. 8. The resistivity decreases with increasing the temperature for all samples, depicting a semiconducting behavior within the 50$290 \mathrm{~K}$ temperature scale. ${ }^{[6]}$ The PNCs obey a resistivity change trend opposite to electrical conductivity. The resistivity is obtained to follow the variable range hopping (VRH) theory, which is related to the network structure in the polymer matrix. To explore the mechanism of electron transportation of the PNCs, the VRH relationship between $\sigma$ and temperature was studied by Equation (5), ${ }^{[68]}$

$$
\sigma=\sigma_{0} \exp \left[-\left(\frac{T_{0}}{T}\right)^{1 /(n+1)}\right], \quad \mathrm{n}=1,2,3
$$

where the constant $T_{0}(\mathrm{~K})$ is the characteristic Mott temperature depending on the electronic wave function localization degree, the constant $\sigma_{0}$ is the electrical conductivity at infinite low temperature, $T(\mathrm{~K})$ is the Kelvin temperature, and $n$ can be equal to 1,2 and 3, meaning for one-, two-, and three- dimensional systems, respectively.

The best linear fits of $\ln (\sigma) \sim T^{-1 /(n+1)}$ for PNCs calculated from Fig. $8 \mathrm{a}$ are presented in Fig. $8 \mathrm{~b}$ with $\mathrm{n}=3$ in a temperature range of $50-290 \mathrm{~K}$, revealing a 3-d electron transport mechanism. $T_{0}$ and $\sigma_{0}$ values can be gained from the intercept and the slope of the plot $\ln (\sigma) \sim T^{-1 /(n+1)}$ and are summarized in Table 3. 

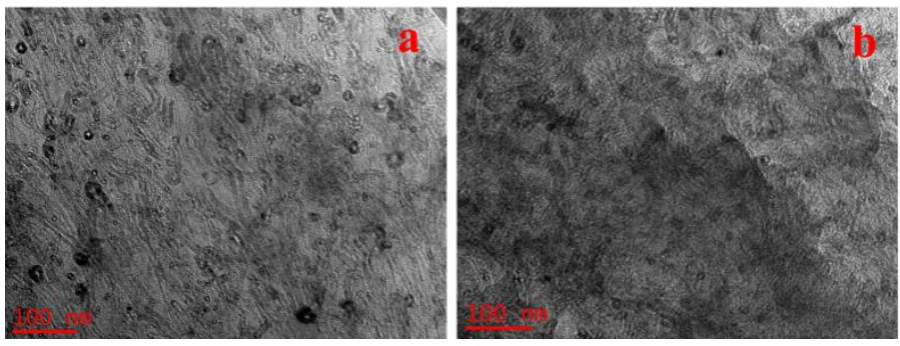

Fig. 7. TEM microstructures of PP/CNT PNCs with (a) $20 \mathrm{~s}$ microwave treatment prepared at $140{ }^{\circ} \mathrm{C}$, (b) $35 \mathrm{~s}$ microwave treatment prepared at $160{ }^{\circ} \mathrm{C}$.

Table 3. The values of paraments $\mathrm{T}_{0}$ and $\sigma_{0}$ for CNTs PNCs.

\begin{tabular}{ccc}
\hline Samples & $\mathrm{T}_{0}(\mathrm{~K})$ & $\sigma_{0}\left(\mathrm{~S} \mathrm{~cm}^{-1}\right)$ \\
\hline $20 \mathrm{~s} \_180{ }^{\circ} \mathrm{C}$ & 43.50 & 0.0058 \\
$30 \mathrm{~s} \_160^{\circ} \mathrm{C}$ & 40.78 & 0.0227 \\
$35 \mathrm{~s} \_140^{\circ} \mathrm{C}$ & 17.02 & 0.0390 \\
$35 \mathrm{~s} \_180^{\circ} \mathrm{C}$ & 22.21 & 0.0486 \\
\hline
\end{tabular}

The variation of the $T_{0}$ and $\sigma_{0}$ values is related to the electrical conductivity mechanism of the composites. The $T_{0}$ value of $\mathrm{PP} / \mathrm{CNT}$ PNCs is observed to obey the order concordant with the measured resistivity values. Generally, a stronger localization of the charge carriers in the system would have a larger $T_{0}$ and thus led to a lower $\sigma^{[69]}$ The $\sigma_{0}$ value increases with elevating CNT loading, and at the same CNTs loading, the $\sigma_{0}$ value is affected by the processing temperature. The $\mathrm{PP} / \mathrm{CNT}$ PNCs with $20 \mathrm{~s}$ microwave heating remains relatively low CNTs, thus the CNTs layer is easily destroyed by $\mathrm{PP}$ at $180{ }^{\circ} \mathrm{C}$. Thus, the charge transport-tation was partitioned which caused a higher $T_{0}$ and lower $\sigma_{0}$.

\subsection{Electronic property of CNTs/PP nanocomposites}

CNTs possess unique properties for promising nanoelectronics application and the bandgap plays an important role in designing CNTs for electronics applications. ${ }^{[70]}$ The optical behavior of pure CNTs and its PNCs was investigated by the ultraviolet-visible (UV-vis) spectra as shown in Fig. 9. The photon-energy $(h v)$ dependence of $\alpha h v$ can be converted from the diffuse reflectance UV-Vis spectra by Tauc's plot, which can be applied for calculating the bandgap $\left(E_{g}\right)$ values as shown by Equation (6), [71]

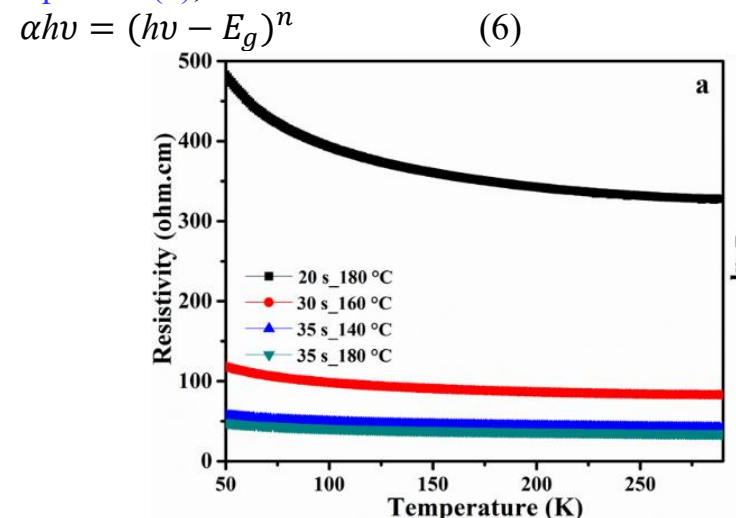

where $\alpha$ is the absorbance coefficient, $h$ is the Planck's constant and $v$ is the photon frequency. The diffuse reflectance UV-vis spectra can be used to convert the Tauc plot. The parameter $n$ is a number depending on different types of electronic transitions. $n=1 / 2$ is for the allowed direct (the minimum energy level of the lowest conduction band positioned directly under the maximum of the highest valence band in $\mathrm{k}$ space), and $n=2$ is for the allowed indirect (the minimum energy level of the lowest conduction band is shifted relative to the maximum of the highest valence band, and the lowest-energy interband transition must then be accompanied by phonon excitation) transitions. ${ }^{[72,73]}$ The reported $n$ is $\sim 2^{[74]}$ for CNTs and the $E_{g}$ was received by extrapolating from the energy axis in the linear portion of the curve. The value $E_{g}$ of pure CNTs is $\sim 1.50 \mathrm{eV}$, which can be compared with the 1.543 $\mathrm{eV}$ reported for the transition between the second pair of van Hove singularity and the Fermi level of CNTs with an index of $(9,8) \cdot{ }^{[75]}$ The $E_{g}$ value of pure PP is lower than that of all PNCs. Meanwhile, the changing trend of $E_{g}$ depends on the $\sigma$ values of the PNCs. The PNCs with a higher $\sigma$ present a lower $E_{g}$. To better study the effects of processing temperature on the $E_{g}$, pure CNTs and the PNCs with $35 \mathrm{~s}$ microwave treatment at different processing temperatures were measured as presented in Fig. 9c. The lower $E_{g}$ is obtained in a certain CNT loading PNCs processed at $160{ }^{\circ} \mathrm{C}$. The changes of $E_{g}$ can be analyzed from the strain of CNTs, which was joined during the hot press process. The reason is that the strain of CNTs could give a positive or negative effect on the electron transportation in CNTs. The uniaxial or torsional strain can result in the insulator-metal transition of SWNT caused by the varied quantum number. ${ }^{[7,, 77]}$ However, because of the elongation of $\mathrm{C}-\mathrm{C}$ bonds, uniaxial strain in graphene leads to the redshift of $2 \mathrm{D}$ and $\mathrm{G}$ band. ${ }^{\left[{ }^{[7]}\right.}$ Moreover, the $E_{g}$ decreases with increasing the CNT loading for the PP/CNTs system as presented in Fig. $9 \mathrm{~d}$. The existing electrons in the delocalized states bring the $\sigma$ in the system. One orbital should be partially filled with electrons. However, the fully filled orbital is inert to obstruct the transport of other electrons. Partially filled states and the state delocalization result in a lower $E_{g}$ due to the CNTs network.

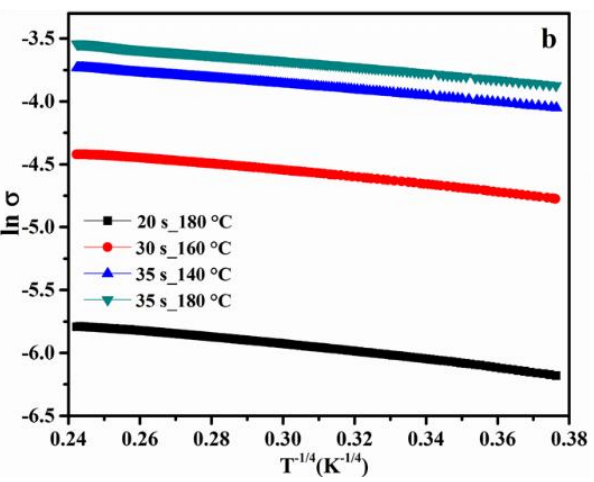

Fig. 8. a) Resistivity $v s$ temperature and b) $\ln (\sigma) v s T^{-1 / 4}$ curves of CNTs PNCs. 

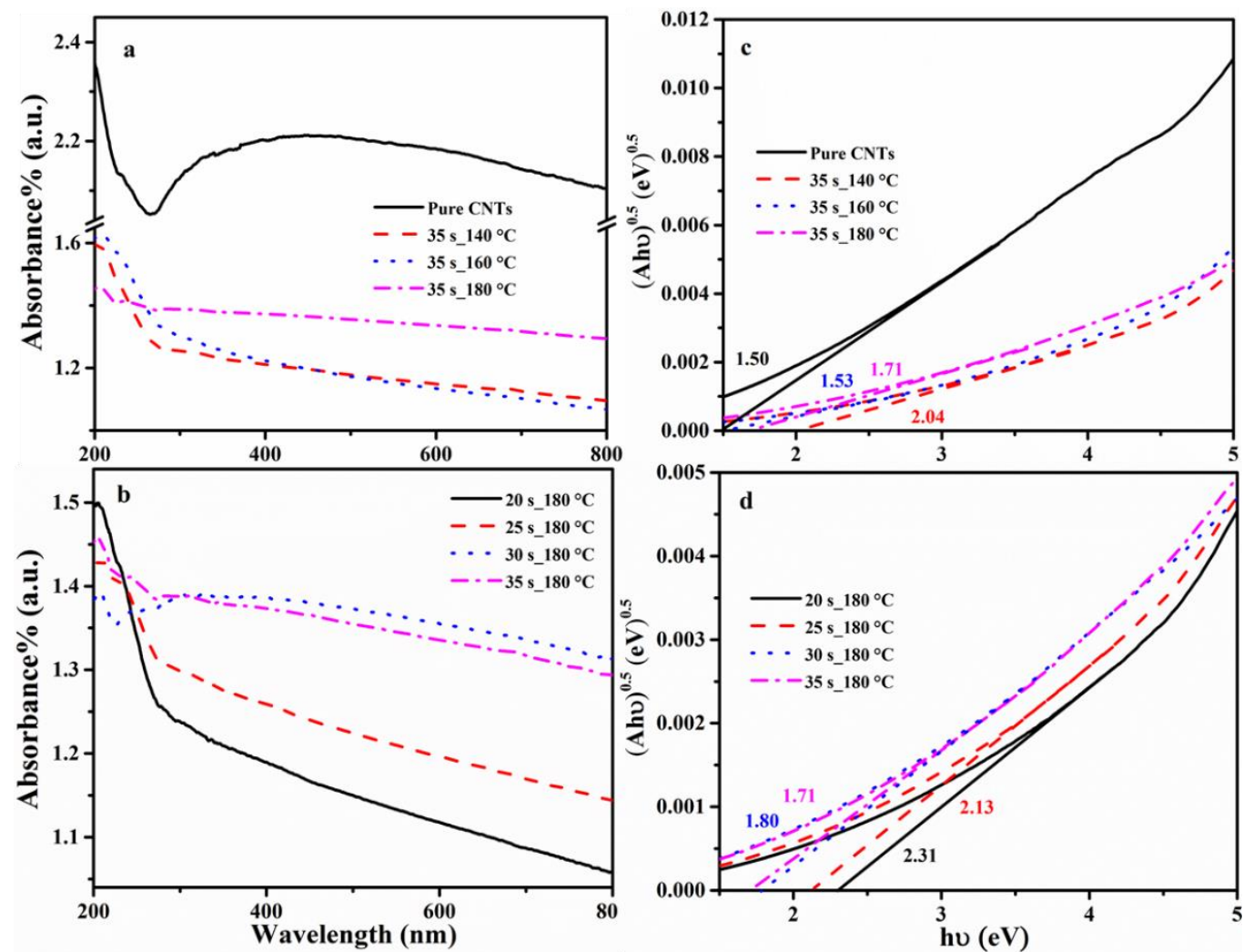

Fig. 9 (a \& b) UV-vis absorbance spectra and corresponding (c \& d) Tauc plots converted from diffuse reflectance spectra data, respectively.

\subsection{Magnetoresistance (MR)}

Fig. 10 presents the magnetoresistance (MR) of PNCs with 30 s microwave treatment processed at $160{ }^{\circ} \mathrm{C}$ measured at 290 $\mathrm{K}$, respectively. Equation (7) can be applied for calculating MR,, 79$]$

$$
\mathrm{MR} \%=[R(H, T)-R(0, T)] / R(0, T) \times 100
$$

where $R(H, T)$ and $R(0, T)$ are the resistance with and without magnetic field $H$, respectively. The trend of MR reveals different magnetic field dependent behaviors that can be divided into positive and negative groups. After applying a magnetic field, the shrinkage in the overlap of electron wave functions causes the positive MR and thus the average length of hopping reduced. ${ }^{[80]}$ The orbital magneto-conductivity theory is utilized to study the negative MR effect, which considers the effects of interference among different hopping paths ${ }^{[81]}$ For PNCs with $30 \mathrm{~s}$ microwave treatment measured at $290 \mathrm{~K}$, a linear negative MR was observed at the low magnetic field. Furthermore, the materials were saturated at high magnetic fields. The ratio of $R(H, T) / R(0, T)$ caused by interference effects is presented as empirical Equation (8)::[82]

$$
\frac{R(H, T)}{R(0, T)} \approx \frac{1}{1+\frac{C_{\text {sat }}\left(H / H_{\text {Sat }}\right)}{1+H / H_{\text {sat }}}}
$$

where the fitting parameters $C_{\text {sat }}$ is a constant and named temperature independent fitting parameter and $H_{\text {sat }}$ is the effective saturation magnetic field. According to the mechanism of Mott VRH electrical conduction, $H_{\text {sat }}$ can be calculated by Equation (9)::[83,84]

$$
H_{\text {sat }} \approx 0.7\left(\frac{8}{3}\right)^{3 / 2}\left(\frac{1}{a_{0}^{2}}\right)\left(\frac{h}{e}\right)\left(\frac{T}{T_{0}}\right)^{3 / 8}
$$

where $h$ is the Planck's constant, and $T_{0}$ is the Mott characteristic temperature $(\mathrm{K})$.

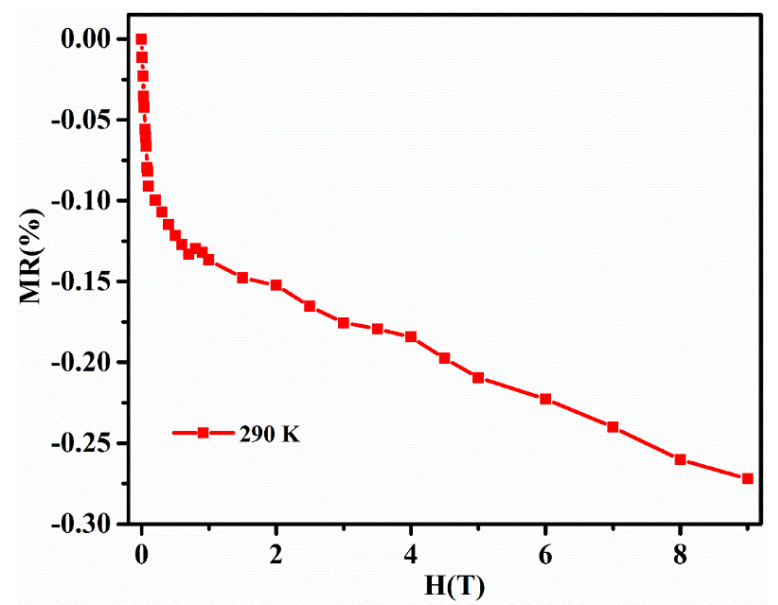

Fig. 10 Magnetoresistance of PNCs with $30 \mathrm{~s}$ microwave treatment processed at $160{ }^{\circ} \mathrm{C}$ characterized at $290 \mathrm{~K}$, respectively.

Equation (9) can be transformed into Equation (10) in the low field limit: 


$$
\frac{R(H, T)}{R(0, T)} \approx 1-C_{\text {sat }} \frac{H}{H_{\text {sat }}}
$$

Substituting Equation (9) into Equation (10) and rearranging, the Equation (11) can calculate MR values:

$$
\begin{aligned}
M R=\frac{R(H, T)-R(0, T)}{R(0, T)} & \approx-C_{\text {sat }}\left[H / H_{\text {sat }}\right]= \\
& -C_{\text {sat }} \frac{H}{0.7\left(\frac{8}{3}\right)^{3 / 2}\left(\frac{1}{a_{0}^{2}}\right)\left(\frac{h}{e}\right)\left(\frac{T}{T_{0}}\right)^{3 / 8}}
\end{aligned}
$$

The calculated $a_{0}$ of PNCs with $1.20 \mathrm{wt} . \%$ CNTs measured at $290 \mathrm{~K}$ are $636.75,495.37$, and $416.120 \mathrm{~nm}$ at $H$ of 2,4 , and $8 \mathrm{~T}$, respectively. Meanwhile, the $a_{0}$ value decreases with increasing the $H$, due to the advance in the polaron population at the expense of bipolarons while the magnetic field is increasing. ${ }^{[85]}$ The negative $\mathrm{MR}$ is also obtained in a nanoporous system composed of bundles of single-walled carbon nanotubes (SWCNTs). ${ }^{[86]}$ However, Yan et al. reported a positive MR obtained from PNCs with 2.6 and $3.0 \mathrm{wt} \%$ CNTs loadings prepared by a solvent surface coating method under the same measurement conditions. ${ }^{\left[{ }^{[6]}\right.}$ The reason caused the difference is that the loadings and network structures of CNTs of the measured samples were affected by two different sample preparation methods. In the same magnetic field, two different electron transport models exist in the corresponding CNTs PNCs, which can be selected depending on desired MR devices.

\section{Conclusions}

Microwave radiation can be used for heating materials selectively. Highly electrically conductive PP/CNT PNCs were successfully synthesized by an efficient microwave solvent-free irradiation method under inert atmosphere and controlled treating time. This method gives a new perspective for designing and producing CNTs based conductive polymer materials on an industrial scale with environment-friendly technology. No effects of microwave radiation on PP granules lasting $35 \mathrm{~s}$ were confirmed. The thermal stability of PNCs gets enhanced by introducing CNTs which was measured under both air and $\mathrm{N}_{2}$ atmospheres. The lower internal chainchain friction heat was obtained in the PNCs than that of pure polymer from melt rheological behaviors, which is caused by the stable interaction between the polymer chains and CNTs. Both XRD and DSC results depicted that higher processing temperate promoted the generation of $\gamma$-phase PP. The morphology study showed that CNTs network structure depended on the processing temperature. Well-formed CNTs network resulted in a higher electrical conductivity and a 3dimension variable range hopping $(\mathrm{VRH})$ was obtained from the electrical conductivity mechanism. The electrical conductivity value of PNCs increased with decreasing the $E_{g}$, which means that the PNCs having a better conductivity prosses a lower $E_{g}$. Negative MR can be obtained in PNCs with $1.20 \mathrm{wt} \%$ CNTs measured at $290 \mathrm{~K}$, providing a favorable approach to design expected MR devices.

\section{Acknowledgments}

This work was financially supported by the University of Tennessee, Knoxville. The authors also would like to thank Ramiz Boy, Jihua Chen, Dale K. Hensley, Yangyang Wang from Oak Ridge National Laboratory for the assistance of some sample tests.

\section{Conflict of Interest}

There is no conflict of interest.

\section{Supporting Information}

Not applicable.

\section{References}

[1] W. Jakes and D. Cox, Microwave mobile communications, Wiley-IEEE Press, 1994.

[2] M. I. Skolnik, Radar Handbook, McGraw-Hill book compaby, 1970.

[3] A. A. Metaxas and R. J. Meredith, Industrial microwave heating, IET, 1983.

[4] R. J. Meredith, Engineers' handbook of industrial microwave heating, IET, 1998.

[5] Q. He, T. Yuan, X. Zhang, X. Yan, J. Guo, D. Ding, M. A. Khan, D. P. Young, A. Khasanov and Z. Luo, J. Phys. Chem. C, 2014, 118, 24784-24796, doi: 10.1021/jp507975r.

[6] E. Thostenson and T. W. Chou, Compos. Part A., 1999, 30, 1055-1071, doi: 10.1016/S1359-835X(99)00020-2.

[7] D. E. Clark, D. C. Folz and J. K. West, Mater. Sci. Eng. A, 2000, 287, 153-158, doi: 10.1016/s0921-5093(00)00768-1.

[8] D. Jiang, V. Murugadoss, Y. Wang, J. Lin, T. Ding, Z. Wang, Q. Shao, C. Wang, H. Liu and N. Lu, Polym. Rev., 2019, 59, 280337, doi: 10.1080/15583724.2018.1546737.

[9] D. K. Agrawal, Curr. Opin. Solid State Mater. Sci., 1998, 3, 480-485, doi: 10.1016/S1359-0286(98)80011-9.

[10] L. Zong, S. Zhou, N. Sgriccia, M. Hawley and L. Kempel, J. Microwave Power Electromagn. Energy, 2003, 38, 49-74, doi: 10.1080/08327823.2003.11688487.

[11] S. Singh, D. Gupta, V. Jain and A. Sharma, Mater. Manuf. Processes, 2015, 30, 1-29, doi: 10.1080/10426914.2014.952028.

[12] X. Guan, G. Q. Zheng, K. Dai, C. Liu, X. Yan, C. Shen and Z. Guo, ACS Appl. Mater. Interfaces, 2016, 8, 14150-14159, doi: 10.1021/acsami.6b02888.

[13] H. Zhao, X. Yan, G. Zhao and Z. Guo, Polym. Eng. Sci., 2016, 8, 939-946, doi: 10.1002/pen.24323.

[14] L. Zhang, Y. Qin, G. Zheng, K. Dai, C. Liu, X. Yan, J. Guo, C. Shen and Z. Guo, Polymer, 2016, 90, 18-25, doi: 10.1016/j.polymer.2016.02.052.

[15] S. Guo, J. Liu, S. Qiu, Y. Wang, X. Yan, N. Wu, S. Wang and Z. Guo, Electrochim. Acta, 2016, 190, 556-565, doi: 10.1016/j.electacta.2015.12.135.

[16] X. Yan, Q. He, X. Zhang, H. Gu, H. Chen, Q. Wang, L. Sun, S. Wei and Z. Guo, Macromol. Mater. Eng., 2014, 299, 485-494, doi: 10.1002/mame.201300208. 
[17] H. Gu, D. Cao, J. Kong, J. Gu, Q. Jiang, Y. Li, B. Wang, X. Yan, Y. Chen and J. E. Ryu, Eng. Sci., 2018, 1, 1-3. doi: $10.30919 / \mathrm{es} 8 \mathrm{~d} 128$.

[18] Y. Liu, M. Shi, C. Yan, Q. Zhuo, H. Wu, L. Wang, H. Liu and Z. Guo, J. Mater. Sci., 2019, 30, 6583-6592, doi: 10.1007/s10854019-00965-2.

[19] H. Wei, C. He, J. Liu, H. Gu, Y. Wang, X. Yan, J. Guo, D. Ding, N. Z. Shen and X. Wang, Polymer, 2015, 67, 192-199, doi: 10.1016/j.polymer.2015.04.064.

[20] H. Gu, J. Guo, X. Zhang, Q. He, Y. Huang, H. A. Colorado, N. Haldolaarachchige, H. Xin, D. P. Young and S. Wei, J. Phys. Chem. C, 2013, 117, 6426-6436, doi: 10.1021/jp311471f.

[21] S. Zhang, H. Liu, S. Yang, X. Shi, D. Zhang, C. Shan, L. Mi, C. Liu, C. Shen and Z. Guo, ACS Appl. Mater. Interfaces, 2019, 11, 10922-10932, doi: 10.1021/acsami.9b00900.

[22] H. Liu, Y. Li, K. Dai, G. Zheng, C. Liu, C. Shen, X. Yan, J. Guo and Z. Guo, J. Mater. Chem. C, 2016, 4, 157-166, doi: 10.1039/C5TC02751A.

[23] L. J. Lee, C. Zeng, X. Cao, X. Han, J. Shen and G. Xu, Compos. Sci. Technol., 2005, 65, 2344-2363, doi: 10.1016/j.compscitech.2005.06.016.

[24] R. Rahimi, M. Ochoa, W. Yu and B. Ziaie, ACS Appl. Mater. Interfaces, 2015, 7, 4463-4470, doi: 10.1021/am509087u.

[25] M. Amjadi, A. Pichitpajongkit, S. Lee, S. Ryu and I. Park, ACS Nano, 2014, 8, 5154-5163, doi: 10.1021/nn501204t.

[26] J. Njuguna and K. Pielichowski, Adv. Eng. Mater., 2003, 5, 769-778, doi: 10.1002/adem.200310101.

[27] A. Nautiyal, M. Qiao, T. Ren, T. Huang, X. Zhang, J. Cook, M. J. Bozack and R. Farag, Eng. Sci., 2018, 4, 70-78, doi: $10.30919 /$ es8d776.

[28] J. Zhu, S. Wei, N. Haldolaarachchige, D. P. Young and Z. Guo, J. Phys. Chem. C, 2011, 115, 15304-15310, doi: 10.1021/jp2052536.

[29] Z. Guo, S. E. Lee, H. Kim, S. Park, H. Hahn, A. Karki and D. Young, Acta Mater, 2009, 57, 267-277, doi: 10.1016/j.actamat.2008.09.024.

[30] L. Lyu, J. Liu, H. Liu, C. Liu, Y. Lu, K. Sun, R. Fan, N. Wang, N. Lu, Z. Guo and E. Wujcik, Eng. Sci., 2018, 2, 26-42, doi: 10.30919/es8d615.

[31] G. Gao, T. Cagin and W. A. Goddard III, Nanotechnology, 1998, 9, 184-191, doi: 10.1088/0957-4484/9/3/007.

[32] X. Wang, Q. Li, J. Xie, Z. Jin, J. Wang, Y. Li, K. Jiang and S. Fan, Nano Lett., 2009, 9, 3137-3141, doi: 10.1021/n1901260b. [33] L. Sun, G. Warren, J. O'reilly, W. Everett, S. Lee, D. Davis, D. Lagoudas and H. J. Sue, Carbon, 2008, 46, 320-328, doi: 10.1016/j.carbon.2007.11.051.

[34] T. Uchida and S. Kumar, J. Appl. Polym. Sci., 2005, 98, $985-$ 989, doi: 10.1002/app.22203.

[35] R. Nie, Q. Wang, P. Sun, R. Wang, Q. Yuan and X. Wang, Eng. Sci., 2019, 6, 22-29, doi: 10.30919/es8d668.

[36] T. Imholt, C. A. Dyke, B. Hasslacher, J. M. Pérez, D. Price, J. A. Roberts, J. Scott, A. Wadhawan, Z. Ye and J. M. Tour, Chem. Mater., 2003, 15, 3969-3970, doi: 10.1021/cm034530g.

[37] P. X. Hou, C. Liu and H. M. Cheng, Carbon, 2008, 46, 2003 2025, doi: 10.1016/j.carbon.2008.09.009.
[38] A. de la Hoz, A. Diaz-Ortiz and A. Moreno, Chem. Soc. Rev., 2005, 34, 164-178, doi: 10.1039/b411438h.

[39] Q. He, T. Yuan, X. Yan, D. Ding, Q. Wang, Z. Luo, T. D. Shen, S. Wei, D. Cao and Z. Guo, Macromol. Chem. Phys., 2014, 215, 327-340, doi: 10.1002/macp.201300608.

[40] X. Yan, M. Li, J. Long, X. Zhang, H. Wei, Q. He, D. Rutman, D. Cao, S. Wei and G. Chen, Macromol. Chem. Phys., 2014, 215, 1098-1106, doi: 10.1002/macp.201400097.

[41] Q. He, T. Yuan, X. Zhang, Z. Luo, N. Haldolaarachchige, L. Sun, D. P. Young, S. Wei and Z. Guo, Macromolecules, 2013, 46, 2357-2368, doi: 10.1021/ma4001397.

[42] A. T. Jones, J. M. Aizlewood and D. Beckett, Macromol. Chem. Phys., 1964, 75, 134-158, doi: 10.1002/macp.1964.020750113.

[43] D. E. Clark and W. H. Sutton, Annu. Rev. Mater. Sci., 1996, 26, 299-331, doi: 10.1146/annurev.ms.26.080196.001503.

[44] J. D. Hoffman, R. L. Miller, H. Marand and D. B. Roitman, Macromolecules, 1992, 25, 2221-2229, doi: 10.1021/ma00034a025.

[45] G. Z. Papageorgiou, D. S. Achilias, D. N. Bikiaris and G. P. Karayannidis, Thermochim. Acta, 2005, 427, 117-128, doi: 10.1016/j.tca.2004.09.001.

[46] H. Tsuji, H. Takai and S. K. Saha, Polymer, 2006, 47, 38263837, doi: 10.1016/j.polymer.2006.03.074.

[47] J. Zhu, S. Wei, Y. Li, L. Sun, N. Haldolaarachchige, D. Young, C. Southworth, A. Khasanov, Z. Luo and Z. Guo, Macromolecules, 2011, 44, 4382-4391, doi: 10.1021/ma102684f.

[48] H. Li and M. A. Huneault, Polymer, 2007, 48, 6855-6866, doi: 10.1016/j.polymer.2007.09.020.

[49] T. Foresta, S. Piccarolo and G. Goldbeck-Wood, Polymer, 2001, 42, 1167-1176, doi: 10.1016/S0032-3861(00)00404-3.

[50] R. Zannetti, G. Celotti, A. Fichera and R. Francesconi, Macromol. Chem. Phys., 1969, 128, 137-142, doi: 10.1002/macp.1969.021280111.

[51] T. Foresta, S. Piccarolo and G. Goldbeck-Wood, Polymer, 2001, 42, 1167-1176, doi: 10.1016/S0032-3861(00)00404-3.

[52] R. Thomann, C. Wang, J. Kressler and R. Mülhaupt, Macromolecules, 1996, 29, 8425-8434, doi: 10.1021/ma951885f.

[53] S. H. Lee, E. Cho, S. H. Jeon and J. R. Youn, Carbon, 2007, 45, 2810-2822, doi: 10.1016/j.carbon.2007.08.042.

[54] S. H. Lee, M. W. Kim, S. H. Kim and J. R. Youn, Eur. Polym. J., 2008, 44, 1620-1630, doi: 10.1016/j.eurpolymj.2008.03.017.

[55] F. Du, R. C. Scogna, W. Zhou, S. Brand, J. E. Fischer and K. I. Winey, Macromolecules, 2004, 37, 9048-9055, doi: $10.1021 / \mathrm{ma} 049164 \mathrm{~g}$.

[56] P. M. Wood-Adams, J. M. Dealy, A. W. Degroot and O. D. Redwine, Macromolecules, 2000, 33, 7489-7499, doi: 10.1021/ma991533z.

[57] A. Tuteja, P. M. Duxbury and M. E. Mackay, Macromolecules, 2007, 40, 9427-9434, doi: 10.1021/ma071313i.

[58] J. Zhang, A. Panwar, D. Bello, T. Jozokos, J. A. Isaacs, C. Barry and J. Mead, Environ. Sci.: Nano, 2016, 3, 409-417, doi: 10.1039/c5en00253b.

[59] A. Wadhawan, D. Garrett and J. M. Pérez, Appl. Phys. Lett., 2003, 83, 2683-2685, doi: 10.1063/1.1615679. 
[60] J. Walkiewicz, G. Kazonich and S. McGill, Miner. Metall. Process, 1988, 5, 39-42, doi: 10.1007/BF03449501.

[61] S. Frank, P. Poncharal, Z. Wang and W. A. de Heer, Science, 1998, 280, 1744-1746, doi: 10.1126/science.280.5370.1744.

[62] Z. Ye, W. D. Deering, A. Krokhin and J. A. Roberts, Phys. Rev. B, 2006, 74, 075425, doi: 10.1103/PhysRevB.74.075425.

[63] M. K. Seo, J. R. Lee and S. J. Park, Mater. Sci. Eng. A, 2005, 404, 79-84, doi: 10.1016/j.msea.2005.05.065.

[64] K. Wu, Y. Xue, W. Yang, S. Chai, F. Chen and Q. Fu, Compos. Sci. Technol., 2016, 130, 28-35, doi: 10.1016/j.compscitech.2016.04.034.

[65] X. Yan, J. Gu, G. Zheng, J. Guo, A. M. Galaska, J. Yu, M. A.

Khan, L. Sun, D. P. Young and Q. Zhang, Polymer, 2016, 103, 315-327, doi: 10.1016/j.polymer.2016.09.056.

[66] A. H. Alshehri, M. Jakubowska, M. Sloma, M. Horaczek, D. Rudka, C. Free and J. D. Carey, Appl. Phys. Lett., 2011, 99, 153109, doi: 10.1063/1.3651278.

[67] J. Stejskal, I. Sapurina, M. Trchová, J. Prokeš, I. Krivka and E. Tobolková, Macromolecules, 1998, 31, 2218-2222, doi: $10.1021 / \mathrm{ma} 970823 \mathrm{~h}$

[68] Z. Guo, K. Shin, A. B. Karki, D. P. Young, R. B. Kaner and H. T. Hahn, J. Nanopart. Res., 2009, 11, 1441-1452, doi: 10.1007/s11051-008-9531-8.

[69] M. Aggarwal, S. Khan, M. Husain, T. Ming, M. Tsai, T. Perng and Z. Khan, Eur. Phys. J. B, 2007, 60, 319-324, doi: 10.1140/epjb/e2007-00348-3.

[70] S. J. Tans, A. R. Verschueren and C. Dekker, Nature, 1998, 393, 49-52, doi: 10.1038/29954.

[71] J. Tauc, Mater. Res. Bull., 1970, 5, 721-729, doi: 10.1016/0025-5408(70)90112-1.

[72] A. Hjelm, C. G. Granqvist and J. M. Wills, Phys. Rev. B, 1996, 54, 2436-2445, doi: 10.1103/PhysRevB.54.2436.

[73] N. Serpone, D. Lawless and R. Khairutdinov, J. Phys. Chem., 1995, 99, 16646-16654, doi: 10.1021/j100045a026.

[74] M. B. Tzolov, T. F. Kuo, D. A. Straus, A. Yin and Xu, J. Phys. Chem. C, 2007, 111, 5800-5804, doi: 10.1021/jp068701r.
[75] J. Wu, W. Walukiewicz, W. Shan, E. Bourret-Courchesne, J. Ager III, K. Yu, E. Haller, K. Kissell, S. M. Bachilo and R. B. Weisman, Phys. Rev. Lett., 2004, 93, 017404, doi: 10.1103/PhysRevLett.93.017404.

[76] O. Gülseren, T. Yildirim, S. Ciraci and Ç. Kılıç, Phys. Rev. B, 2002, 65, 155410, doi: 10.1103/PhysRevB.65.155410.

[77] L. Yang, M. P. Anantram, J. Han and J. P. Lu, Phys. Rev. B, 1999, 60, 13874-13878, doi: 10.1103/PhysRevB.60.13874.

[78] Z. H. Ni, T. Yu, Y. H. Lu, Y. Y. Wang, Y. P. Feng and Z. X. Shen, ACS Nano, 2008, 2, 2301-2305, doi: 10.1021/nn800459e.

[79] B. Hu and Y. Wu, Nat. Mater, 2007, 6, 985-991, doi: $10.1038 /$ nmat2034.

[80] T. A. Skotheim and J. Reynolds, Handbook of Conducting Polymers, CRC press, 2007.

[81] J. Guo, H. Gu, H. Wei, Q. Zhang, N. Haldolaarachchige, Y. Li, D. P. Young, S. Wei and Z. Guo, J. Phys. Chem. C, 2013, 117, 10191-10202, doi: 10.1021/jp402236n.

[82] A. J. Hoffman, L. Alekseyev, S. S. Howard, K. J. Franz, D. Wasserman, V. A. Podolskiy, E. E. Narimanov, D. L. Sivco and C. Gmachl, Nat. Mater, 2007, 6, 946-950, doi: 10.1038/nmat2033.

[83] T. I. Su, C. R. Wang, S. T. Lin and R. Rosenbaum, Phys. Rev. $B, 2002$, 66, 054438, doi: 10.1103/PhysRevB.66.054438.

[84] A. Sarkar, P. Ghosh, A. K. Meikap, S. K. Chattopadhyay, S. K. Chatterjee and M. Ghosh, Solid State Commun., 2007, 143, 358-363, doi: 10.1016/j.ssc.2007.05.013.

[85] P. Bobbert, T. Nguyen, W. Wagemans, F. van Oost, B. Koopmans and M. Wohlgenannt, Synth. Met., 2010, 160, 223-229, doi: 10.1016/j.synthmet.2009.06.002.

[86] Y. Yosida and I. Oguro, J. Appl. Phys., 1999, 86, 999-1003, doi: 10.1063/1.370838.

Publisher's Note Engineered Science Publisher remains neutral with regard to jurisdictional claims in published maps and institutional affiliations. 